\title{
STRUCTURE-ACTIVITY STUDIES OF 20-DEOXO-20-AMINO DERIVATIVES OF TYLOSIN-RELATED MACROLIDES
}

\author{
H. A. Kirst, K. E. Willard, M. Debono, J. E. Toth, B. A. Truedell, \\ J. P. Leeds, J. L. Ott, A. M. Felty-Duckworth and F. T. Counter \\ Lilly Research Laboratories, Eli Lilly and Company, \\ Indianapolis, Indiana 46285, U.S.A. \\ E. E. Ose, G. D. Crouse and J. M. Tustin \\ Lilly Research Laboratories, Eli Lilly and Company, \\ Greenfield, Indiana 46140, U.S.A. \\ SATOSHI ŌMURA
}

The Kitasato Institute, and School of Pharmaceutical Sciences, Kitasato University, Minato-ku, Tokyo 108, Japan

(Received for publication June 21, 1989)

\begin{abstract}
Reductive amination of the $\mathrm{C}-20$ aldehyde group of tylosin and related macrolides yielded a large series of derivatives with potentially useful antibiotic properties. Evaluation of these new compounds was conducted on the basis of: 1) Broad antimicrobial spectrum in vitro, with particular emphasis on inhibition of Pasteurella multocida and Pasteurella haemolytica; 2) in vivo efficacy, especially when given orally, against $P$. multocida in experimental infections in chicks; and 3) bioavailability after oral administration to laboratory animals. The most useful activity was found within a series of derivatives produced by reductive amination of desmycosin with secondary amines.
\end{abstract}

Tylosin is an important and well-established antibiotic in veterinary medicine ${ }^{1)}$; it is orally effective against infections in poultry and pigs due to Gram-positive bacteria and Mycoplasma gallisepticum as well as certain Gram-negative bacteria such as Fusobacterium necrophorum, a causative agent of liver abscesses in cattle $\mathrm{e}^{2)}$. A variety of chemical modifications of the $\mathrm{C}-20$ aldehyde group in tylosin and related macrolide antibiotics have recently been reported, which improved efficacy against experimental infections and bioavailability in animals after oral administration ${ }^{3}$. From our extensive evaluation of several series of macrolide derivatives, potentially useful activity was found in a compound obtained by reductive amination of desmycosin, which resulted in an expansion of the effective antimicrobial spectrum of tylosin to include both Pasteurella multocida and Pasteurella haemolytica ${ }^{4}$. These Gram-negative bacteria are important pathogens responsible for respiratory illness and mortality in both cattle and pigs ${ }^{5,6)}$. Since a macrolide antibiotic which could be administered both parenterally and orally for the treatment of infections caused by Pasteurella species would be a useful contribution to veterinary medicine, an extensive exploration of the structure-activity relationships among reductively aminated derivatives of macrolides was warranted. In this paper, we report the results of our studies on numerous such derivatives of tylosin-related macrolides.

\section{Results and Discussion}

\section{Synthesis of Reductive Amination Derivatives}

The effects of reductive amination of the aldehyde group in 16-membered macrolide antibiotics have 
been only recently investigated ${ }^{7)}$. ŌMURA et al., reported that 20-deoxo-20-aminotylosin was a very poor antibiotic, but a dimeric derivative (20,20' -dideoxo-20,20'-iminoditylosin) had good antimicrobial activity ${ }^{8}$. In a subsequent study, reductive amination of tylosin and desmycosin was performed with a variety of primary and secondary amines, yielding a series of secondary and tertiary amino derivatives; several of these compounds demonstrated good antimicrobial activity both in vitro and in vivo against Gram-positive bacteria $^{9}$.

During this period, a new series of derivatives of 5-O-mycaminosyltylonolide (OMT) was reported, in which activity against Gram-negative bacteria was significantly increased when the $\mathrm{C}-23$ substituent of OMT was converted into certain tertiary amino groups ${ }^{10}$. Our reductive amination of desmycosin or lactenocin with hexamethyleneimine (one of the C-23 amino substituents which increased activity of OMT ${ }^{10)}$ yielded new macrolide derivatives which exhibited activity against $P$. multocida both in vitro and in vivo ${ }^{4}$. This unexpected and novel result prompted our synthesis of a large number of reductively aminated derivatives in order to define the structure-activity relationships and to determine whether a suitable candidate for use in veterinary medicine could be identified.

Standard reductive amination procedures employing sodium cyanoborohydride proved very suitable for the synthesis of a wide variety of derivatives (Scheme 1$)^{11)}$. Selective modification of the aldehyde function was easily accomplished under these mild conditions, with no interference from side reactions such as reduction of the C-9 ketone. Derivatives were prepared from both primary and secondary amines, but the latter were emphasized because of the superior antimicrobial properties of the tertiary amino derivatives (see below). Also, as a result of our initial antimicrobial evaluation, we prepared a greater number of derivatives which possessed aliphatic $N$-alkyl groups in which the total number of carbon atoms of the amino substituents ranged from six to about fourteen. Reductive aminations were conducted using both acyclic and cyclic secondary amines and incorporated both unsaturation and hetero atom substitutions

Scheme 1. Reaction scheme and structures of reductively aminated derivatives of tylosin-related macrolides.

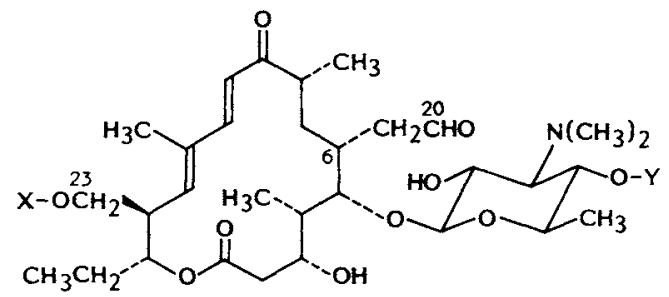

Macrolides used as starting materials for reductive aminations

\begin{tabular}{|c|c|c|}
\hline $\mathrm{X}$ & $\mathrm{Y}=$ Mycarosyl & $\mathrm{Y}=\mathrm{H}$ \\
\hline Mycinosyl & Tylosin & Desmycosin \\
\hline $\begin{array}{l}\text { 3-O-Demethyl- } \\
\text { mycinosyl }\end{array}$ & Macrocin & Lactenocin \\
\hline Allosyl & DOMM & DOML \\
\hline $\mathrm{H}$ & DMT & OMT \\
\hline
\end{tabular}

$\mathrm{DMT}=$ Demycinosyltylosin

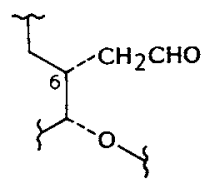

$$
\frac{\mathrm{R}_{1} \mathrm{R}_{2} \mathrm{NH}, \mathrm{NaBH}_{3} \mathrm{CN}}{\text { MeOH, room temp }}
$$<smiles>[3H]CCCC(CC[12CH2])C([3H])O[3H]</smiles> 
Table 1. Structure and physico-chemical data for derivatives from reductive amination of desmycosin.

\begin{tabular}{|c|c|c|c|c|c|}
\hline \multirow{2}{*}{ Compound } & \multicolumn{2}{|c|}{ Substituents from Scheme 1} & \multirow{2}{*}{$\mathrm{FD}-\mathrm{MS}^{\mathrm{a}}$} & \multirow{2}{*}{$\begin{array}{c}\text { UV } \\
\lambda_{\max } \operatorname{nm}(\varepsilon)\end{array}$} & \multirow{2}{*}{ NMR of $N R_{1} R_{2}{ }^{b}$} \\
\hline & $\mathbf{R}_{1}$ & $\mathbf{R}_{2}$ & & & \\
\hline 1 & $\mathrm{H}$ & $\mathrm{H}$ & 773 & $284(15,200)$ & Exchangeable $\mathrm{H}$ \\
\hline 2 & $\mathbf{H}$ & Methyl & 787 & $284(17,900)$ & $2.49\left(3 \mathrm{H}, \mathrm{s}, \mathrm{NCH}_{3}\right)$ \\
\hline 3 & $\mathrm{H}$ & tert-Butyl & 829 & $284(22,800)$ & $1.08(9 \mathrm{H}, \mathrm{s}$, tert-butyl $)$ \\
\hline 4 & $\mathrm{H}$ & Neopentyl & 843 & $282(29,270)$ & $0.96(9 \mathrm{H}, \mathrm{s}$, tert-butyl $)$ \\
\hline 5 & $\mathrm{H}$ & $n$-Hexyl & 857 & $283(21,840)$ & $0.95\left(3 \mathrm{H}, \mathrm{t}, \mathrm{CH}_{3}\right)$ \\
\hline 6 & $\mathrm{H}$ & Benzyl & 863 & $281(20,900)$ & $7.25 \sim 7.35(5 \mathrm{H}, \mathrm{m}$, phenyl $)$ \\
\hline 7 & $\mathrm{H}$ & 2-Ph-ethyl & 877 & $283(22,900)$ & $7.25 \sim 7.40(5 \mathrm{H}, \mathrm{m}$, phenyl $)$ \\
\hline 8 & $\mathrm{H}$ & 1-Adamantyl & 907 & $283(21,500)$ & $1.5 \sim 2.5$ (complex multiplets) \\
\hline 9 & $\mathrm{H}$ & 2-Adamantyl & 907 & $282(20,250)$ & $1.5 \sim 2.5$ (complex multiplets) \\
\hline 10 & $\mathrm{H}$ & $n$-Octyl & 885 & $282(11,000)$ & $0.95\left(3 \mathrm{H}, \mathrm{t}, \mathrm{CH}_{3}\right)$ \\
\hline 11 & Methyl & Methyl & 801 & $283(19,550)$ & $2.15\left(6 \mathrm{H}, \mathrm{s}, \mathrm{NCH}_{3}\right)$ \\
\hline 12 & Ethyl & Ethyl & 829 & $283(22,410)$ & $1.05\left(6 \mathrm{H}, \mathrm{t}, \mathrm{CH}_{3}\right)$ \\
\hline 13 & Propyl & Propyl & 857 & $282(18,370)$ & $0.95\left(6 \mathrm{H}, \mathrm{t}, \mathrm{CH}_{3}\right)$ \\
\hline 14 & iso-Propyl & iso-Propyl & 857 & $281(21,730)$ & $1.0\left(12 \mathrm{H}, \mathrm{d}, \mathrm{CH}_{3}\right)$ \\
\hline 15 & $n$-Butyl & $n$-Butyl & 885 & $282(20,160)$ & $0.95\left(6 \mathrm{H}, \mathrm{t}, \mathrm{CH}_{3}\right)$ \\
\hline 16 & iso-Butyl & iso-Butyl & 885 & $280(21,770)$ & $0.90\left(12 \mathrm{H}, \mathrm{m}, \mathrm{CH}_{3}\right)$ \\
\hline 17 & sec-Butyl & sec-Butyl & 885 & $280(22,040)$ & $0.9 \sim 1.0\left(12 \mathrm{H}, \mathrm{m}, \mathrm{CH}_{3}\right)$ \\
\hline 18 & Ethyl & sec-Butyl & 857 & $282(22,000)$ & $0.9\left(9 \mathrm{H}, \mathrm{m}, \mathrm{CH}_{3}\right)$ \\
\hline 19 & Ethyl & 2-Me-butyl & 871 & $282(23,000)$ & $0.9\left(9 \mathrm{H}, \mathrm{m}, \mathrm{CH}_{3}\right)$ \\
\hline 20 & Ethyl & Neopentyl & 871 & $282(22,000)$ & $0.86(9 \mathrm{H}, \mathrm{s}$, tert -butyl $)$ \\
\hline 21 & $n$-Hexyl & $n$-Hexyl & 941 & $283(22,000)$ & $0.9\left(6 \mathrm{H}, \mathrm{t}, \mathrm{CH}_{3}\right), 1.3\left(\mathrm{CH}_{2}\right)$ \\
\hline 22 & Cyclohexyl & Cyclohexyl & 937 & $282(16,900)$ & $1.5 \sim 1.9(\mathrm{~m}$, aliphatic) \\
\hline 23 & Benzyl & Benzyl & 953 & $281(20,320)$ & $7.2 \sim 7.4(10 \mathrm{H}, \mathrm{m}$, phenyl $)$ \\
\hline 24 & n-Octyl & Norbornyl & 979 & $281(21,000)$ & 1.2 (br s, aliphatic) \\
\hline 25 & $n-\mathrm{C}_{12} \mathrm{H}_{25}$ & Norbornyl & 1,035 & $282(19,000)$ & 1.3 (br s, aliphatic) \\
\hline 26 & $n-\mathrm{C}_{12} \mathrm{H}_{25}$ & Cyclooctyl & 1,051 & $282(20,000)$ & 1.2 (br s, aliphatic) \\
\hline 27 & Allyl & Allyl & 853 & $283(22,000)$ & $5.2(4 \mathrm{H}, \mathrm{m}), 5.7(2 \mathrm{H}, \mathrm{m})$ \\
\hline 28 & Allyl & 2-Cl-allyl & 887,888 & $281(22,000)$ & $\begin{array}{l}5.0 \sim 5.3(4 \mathrm{H}, \mathrm{m}), 5.7(1 \mathrm{H}, \mathrm{m}) \\
3.2(2 \mathrm{H}, \mathrm{d})\end{array}$ \\
\hline 29 & Allyl & $2-\mathrm{Br}-\mathrm{allyl}$ & 930,932 & $282(21,000)$ & $\begin{array}{l}5.0 \sim 5.2(2 \mathrm{H}, \mathrm{m}), 5.55 \text { and } 5.77 \\
(1 \mathrm{H} \text { each, br s }), 5.7(1 \mathrm{H}, \mathrm{m})\end{array}$ \\
\hline 30 & $n$-Butyl & Methallyl & 882 & $282(22,000)$ & $4.7(2 \mathrm{H}, \mathrm{d}), 2.6(2 \mathrm{H}, \mathrm{t})$ \\
\hline 31 & iso-Butyl & Allyl & 868 & $281(22,000)$ & $5.1(\mathrm{~m}), 4.7(\mathrm{~m})$ \\
\hline 32 & Propargyl & Propargyl & 848 & $283(19,000)$ & $2.3(2 \mathrm{H}, \mathrm{s}, \mathrm{CH})$ \\
\hline 33 & Propargyl & iso-Butyl & 866 & $282(17,000)$ & $0.9\left(3 \mathrm{H}, \mathrm{d}, \mathrm{CH}_{3}\right)$ \\
\hline 34 & $1-\mathrm{Me}_{3} \mathrm{Si}$-propargyl & n-Propyl & 925 & $281(17,000)$ & $\begin{array}{l}0.16(9 \mathrm{H}, \mathrm{s}, \mathrm{TMS}) \\
0.9\left(3 \mathrm{H}, \mathrm{t}, \mathrm{CH}_{3}\right)\end{array}$ \\
\hline 35 & Phenyl & Phenyl & 925 & $282(28,500)$ & $6.9 \sim 7.35(10 \mathrm{H}, \mathrm{m}$, phenyl $)$ \\
\hline & & & & $250(13,200)$ & \\
\hline \multicolumn{6}{|c|}{$\mathrm{NR}_{1} \mathrm{R}_{2}$} \\
\hline 36 & \multicolumn{2}{|l|}{ Morpholinyl } & 843 & $284(20,400)$ & $3.7 \sim 3.8(4 \mathrm{H}, \mathrm{m}, \alpha$ to oxygen $)$ \\
\hline 37 & \multicolumn{2}{|l|}{ Thiomorpholinyl } & 859 & $283(20,730)$ & $2.7(4 \mathrm{H}, \mathrm{m}, \alpha$ to nitrogen $)$ \\
\hline 38 & \multicolumn{2}{|l|}{ Piperidinyl } & 841 & $283(24,500)$ & $1.5 \sim 1.7$ and 2.3 (aliphatic) \\
\hline 39 & \multicolumn{2}{|c|}{ 3,5-Di-Me-morpholinyl } & 871 & $282(20,500)$ & 1.13 and $1.3\left(3 \mathrm{H} \mathrm{each}, \mathrm{d}, \mathrm{CH}_{3}\right)$ \\
\hline 40 & \multicolumn{2}{|c|}{ 3,5-Di-Me-piperidinyl } & 869 & $283(22,570)$ & 0.80 and $1.00\left(3 \mathrm{H}\right.$ each, $\left.\mathrm{CH}_{3}\right)$ \\
\hline 41 & \multicolumn{2}{|l|}{ 4-Ph-piperazinyl } & 917 & $\begin{array}{l}283(13,700) \\
248(31,200)\end{array}$ & $6.92 \sim 7.35(5 \mathrm{H}, \mathrm{m}$, phenyl $)$ \\
\hline 42 & \multicolumn{2}{|l|}{ 4-Ph-piperidinyl } & 917 & $\begin{array}{l}283(21,700) \\
205(14,800)\end{array}$ & $7.25 \sim 7.4(5 \mathrm{H}, \mathrm{m}$, phenyl $)$ \\
\hline 43 & \multirow{2}{*}{\multicolumn{2}{|c|}{$\begin{array}{l}\text { 4-Ph-1,2,3,6-tetrahydropyridinyl } \\
\text { Thiazolidinyl }\end{array}$}} & 915 & $282(21,000)$ & $6.05(1 \mathrm{H}, \mathrm{brs}), 7.1 \sim 7.4(5 \mathrm{H}, \mathrm{n}$ \\
\hline 44 & & & 845 & $282(21,800)$ & $5.3\left(2 \mathrm{H}, \mathrm{s}, \mathrm{NCH}_{2} \mathrm{~S}\right)$ \\
\hline
\end{tabular}

a Parent ion in FD-MS.

b Chemical shifts in $\delta(\mathrm{ppm})$ for characteristic protons of dialkylamino substituent; C-20 aldehyde proton from starting macrolide absent. 
Table 2. Structure and physico-chemical data for derivatives from reductive amination of tylosin and related macrolides.

\begin{tabular}{|c|c|c|c|c|c|c|}
\hline \multirow{2}{*}{ Compound } & \multicolumn{2}{|c|}{ Substituents from Scheme 1} & \multirow{2}{*}{ Parent } & \multirow{2}{*}{ FD-MS ${ }^{a}$} & \multirow{2}{*}{$\begin{array}{c}\mathrm{UV} \\
\lambda_{\max } \operatorname{nm}(\varepsilon)\end{array}$} & \multirow{2}{*}{$\mathrm{NMR}$ of $\mathrm{NR}_{1} \mathrm{NR}_{2}{ }^{\mathrm{b}}$} \\
\hline & $\mathrm{R}_{1}$ & $\mathrm{R}_{2}$ & & & & \\
\hline 45 & $\mathrm{H}$ & Methyl & Tylosin & 912 & $283(19,900)$ & $2.43\left(3 \mathrm{H}, \mathrm{s}, \mathrm{NCH}_{3}\right)$ \\
\hline 46 & $\mathrm{H}$ & Benzyl & Tylosin & 1,006 & $283(20,300)$ & $7.3 \sim 7.4(5 \mathrm{H}, \mathrm{m}$, phenyl $)$ \\
\hline 47 & $\mathrm{H}$ & 2-Ph-ethyl & Tylosin & 1,021 & $282(23,100)$ & $7.25 \sim 7.35(5 \mathrm{H}, \mathrm{m}$, phenyl $)$ \\
\hline 48 & Methyl & Methyl & Tylosin & 945 & $285(19,800)$ & $2.47\left(6 \mathrm{H}, \mathrm{s}, \mathrm{NCH}_{3}\right)$ \\
\hline 49 & Benzyl & Benzyl & Tylosin & 1,095 & $283(17,300)$ & $7.24 \sim 7.4(10 \mathrm{H}, \mathrm{m}$, phenyl) \\
\hline 50 & Benzyl & Benzyl & DOMM & 1,069 & $281(21,300)$ & $7.2 \sim 7.45(10 \mathrm{H}, \mathrm{m}$, phenyl $)$ \\
\hline 51 & Benzyl & Benzyl & DOML & 924 & $282(20,700)$ & $7.2 \sim 7.45(10 \mathrm{H}, \mathrm{m}$, phenyl $)$ \\
\hline 52 & $\mathrm{H}$ & Methyl & OMT & 613 & $285(18,900)$ & $2.39\left(3 \mathrm{H}, \mathrm{s}, \mathrm{NCH}_{3}\right)$ \\
\hline 53 & $\mathrm{H}$ & Benzyl & OMT & 689 & $285(17,400)$ & $7.25 \sim 7.35(5 \mathrm{H}, \mathrm{m}$, phenyl $)$ \\
\hline 54 & $\mathbf{H}$ & 2-Ph-ethyl & OMT & 703 & $282(18,800)$ & $7.25 \sim 7.35(5 \mathrm{H}, \mathrm{m}$, phenyl $)$ \\
\hline 55 & Methyl & Methyl & OMT & 627 & $284(19,900)$ & $2.36\left(6 \mathrm{H}, \mathrm{s}, \mathrm{NCH}_{3}\right)$ \\
\hline \multirow[t]{2}{*}{56} & Phenyl & Phenyl & OMT & 750 & $\begin{array}{l}282(26,800) \\
250(13,300)\end{array}$ & $6.9 \sim 7.4(10 \mathrm{H}, \mathrm{m}$, phenyl $)$ \\
\hline & \multicolumn{2}{|c|}{$\mathrm{NR}_{1} \mathrm{R}_{2}$} & & & & \\
\hline 57 & \multicolumn{2}{|c|}{ Heptamethyleneimino } & Tylosin & 1,013 & $282(22,000)$ & 1.6 and 2.5 (aliphatic) \\
\hline 58 & \multicolumn{2}{|c|}{ 3-Azabicyclo[3.2.2]nonanyl } & Tylosin & 1,025 & $282(21,500)$ & $1.6 \sim 1.9$ and $2.4 \sim 2.6$ (aliphatic) \\
\hline 59 & \multicolumn{2}{|c|}{ Morpholinyl } & Tylosin & 985 & $283(20,500)$ & $3.7(4 \mathrm{H}, \mathrm{m}, \alpha$ to oxygen $)$ \\
\hline 60 & \multicolumn{2}{|c|}{ Hexamethyleneimino } & Macrocin & 985 & $283(20,400)$ & 1.6 and 2.5 (aliphatic) \\
\hline 61 & \multicolumn{2}{|c|}{ Morpholinyl } & Macrocin & 973 & $283(22,700)$ & $3.7(4 \mathrm{H}, \mathrm{m}, \alpha$ to oxygen $)$ \\
\hline 62 & \multicolumn{2}{|c|}{ Heptamethyleneimino } & Lactenocin & 855 & $282(21,500)$ & 1.6 and 2.5 (aliphatic) \\
\hline 63 & \multicolumn{2}{|c|}{ Morpholinyl } & Lactenocin & 829 & $284(19,800)$ & $3.7(4 \mathrm{H}, \mathrm{m}, \alpha$ to oxygen $)$ \\
\hline 64 & \multicolumn{2}{|c|}{ Hexamethyleneimino } & OMT & 681 & $284(19,700)$ & 1.6 and 2.5 (aliphatic) \\
\hline 65 & \multicolumn{2}{|c|}{ 4-Ph-piperidinyl } & OMT & 743 & $284(19,600)$ & $7.2 \sim 7.45(5 \mathrm{H}, \mathrm{m}$, phenyl $)$ \\
\hline 66 & \multicolumn{2}{|c|}{ 3,5-Dimethylpiperidinyl } & OMT & 695 & $284(20,400)$ & 0.80 and $1.0\left(3 \mathrm{H}, \mathrm{d}, \mathrm{CH}_{3}\right)$ \\
\hline 67 & \multicolumn{2}{|c|}{ Morpholinyl } & OMT & 669 & $283(19,500)$ & $2.5(\alpha$ to $\mathrm{N}), 3.7(\alpha$ to $\mathrm{O})$ \\
\hline 68 & \multicolumn{2}{|c|}{ 3,5-Dimethylmorpholinyl } & OMT & 696 & $284(21,400)$ & $1.15,1.33\left(3 \mathrm{H}\right.$ each, $\left.\mathrm{CH}_{3}\right)$ \\
\hline 69 & \multicolumn{2}{|c|}{ 3-Azabicyclo[3.2.2]nonanyl } & OMT & 707 & $282(19,000)$ & $1.6 \sim 2.0$ and $2.4 \sim 2.6$ (aliphatic) \\
\hline 70 & \multicolumn{2}{|l|}{ Tylosin ${ }^{\mathrm{c}}$} & & 915 & $282(24,500)$ & $9.68(1 \mathrm{H}, \mathrm{s}$, aldehyde $)$ \\
\hline 71 & \multicolumn{2}{|c|}{ Desmycosin ${ }^{c}$} & & 771 & $283(19,200)$ & $9.69(1 \mathrm{H}, \mathrm{s}$, aldehyde $)$ \\
\hline 72 & \multicolumn{2}{|l|}{$\mathrm{OMT}^{\mathrm{c}}$} & & 597 & $283(20,500)$ & $9.70(1 \mathrm{H}, \mathrm{s}$, aldehyde $)$ \\
\hline
\end{tabular}

$a, b$ See footnotes in Table 1.

c Reference standard.

within the amino substituents. Approximately one hundred reductive amination derivatives of tylosin-related macrolides have been prepared and evaluated. Additional details of the synthesis and evaluation of one particular group of derivatives, prepared from desmycosin and cyclic secondary amines, have been recently reported $^{12)}$

The new compounds were isolated by standard extractive procedures and purified by precipitation from aqueous acidic solution upon addition of aqueous sodium hydroxide; when necessary, further purification was accomplished by chromatographic methods ${ }^{12)}$. The new products were fully characterized by their ${ }^{1} \mathrm{H}$ NMR, IR, UV and MS (Tables I and 2).

Evaluation of Reductive Amination Derivatives of Desmycosin: In Vitro Activity

All of the derivatives which were prepared by reductive amination of tylosin, desmycosin and related 16-membered macrolides exhibited some antimicrobial activity in vitro (Table 3). Representative pathogens from both clinical and veterinary medicine were employed in the initial in vitro screening tests. Derivatives with a primary amino group at C-20 (compound $\mathbf{1}$ ) or possessing one or two methyl groups on the amino 
Table 3. In vitro antimicrobial activity of derivatives from reductive amination of tylosin and related macrolides.

\begin{tabular}{|c|c|c|c|c|c|c|c|c|c|c|}
\hline \multirow{2}{*}{ Compound } & \multicolumn{10}{|c|}{$\operatorname{MIC}(\mu \mathrm{g} / \mathrm{ml})^{\mathrm{a}}$} \\
\hline & S. a. & S. py. & S. pn. & $H . i$ & P. $m$. & $P . h$ & $M . g$. & $M . s$ & M. hr. & M. hp. \\
\hline 1 & 8 & 32 & 32 & 128 & $>50$ & $>50$ & 6.2 & 25 & $>50$ & 25 \\
\hline 2 & 16 & 4 & 64 & $>128$ & $>50$ & $>50$ & 12.5 & 12.5 & $>50$ & 25 \\
\hline 3 & 8 & 2 & 16 & 64 & 50 & $>50$ & 6.2 & 3.1 & 25 & 3.1 \\
\hline 4 & 1 & 0.5 & 4 & 16 & 12.5 & 12.5 & 3.1 & 0.4 & 25 & 1.6 \\
\hline 5 & 1 & 0.5 & 1 & 8 & 6.2 & 12.5 & 0.4 & 0.4 & 50 & 3.1 \\
\hline 6 & 1 & 0.2 & 0.5 & 8 & 6.2 & 6.2 & 0.8 & 0.2 & 50 & $\mathrm{NG}$ \\
\hline 7 & 0.5 & 0.2 & 1 & 8 & 3.1 & 3.1 & 0.8 & 0.4 & $>50$ & 0.8 \\
\hline 8 & 1 & 0.2 & 2 & 16 & 6.2 & 12.5 & 0.4 & 0.4 & 50 & 6.2 \\
\hline 9 & 0.5 & 0.2 & 0.5 & 8 & 3.1 & 6.2 & 0.1 & 0.1 & 50 & 1.6 \\
\hline 10 & 1 & 0.1 & 0.5 & 16 & 12.5 & 6.2 & 0.4 & 0.4 & 25 & 3.1 \\
\hline 11 & 4 & 2 & 4 & 16 & 50 & 50 & 12.5 & 6.2 & $>50$ & 12.5 \\
\hline 12 & 2 & 0.5 & 1 & 16 & 25 & 25 & 6.2 & 1.6 & 50 & 12.5 \\
\hline 13 & I & 0.2 & 1 & 16 & 6.2 & 6.2 & 1.6 & 0.8 & 12.5 & 0.8 \\
\hline 14 & 2 & 1 & 4 & 32 & 25 & 50 & 6.2 & 1.6 & $>50$ & 12.5 \\
\hline 15 & 0.5 & 0.2 & 1 & 16 & 12.5 & 6.2 & 0.8 & 0.2 & 25 & 0.8 \\
\hline 16 & 1 & 0.2 & 1 & 16 & 6.2 & 6.2 & 0.4 & 0.2 & 25 & 0.8 \\
\hline 17 & 0.5 & 0.2 & 0.5 & 8 & 12.5 & 12.5 & 0.8 & 0.4 & 25 & 0.8 \\
\hline 18 & 2 & 0.5 & 2 & 64 & 12.5 & 12.5 & 0.2 & 0.2 & 12.5 & 0.8 \\
\hline 19 & I & 0.2 & 0.5 & 8 & 12.5 & 6.2 & $<0.1$ & 0.4 & 12.5 & 0.8 \\
\hline 20 & I & 0.2 & 0.2 & 4 & 12.5 & 12.5 & 0.8 & 0.4 & 6.2 & 0.4 \\
\hline 21 & 1 & 0.2 & 0.5 & 8 & 12.5 & 12.5 & NG & NG & $\mathrm{NG}$ & 1.6 \\
\hline 22 & 1 & 0.2 & 0.2 & 16 & 6.2 & 6.2 & 0.4 & 0.2 & 50 & 0.4 \\
\hline 23 & 0.5 & 0.2 & 0.5 & 32 & 12.5 & 6.2 & $<0.1$ & 0.2 & 6.2 & 1.6 \\
\hline 24 & 1 & 0.5 & 1 & 32 & 25 & 25 & NG & 0.2 & 6.2 & 0.1 \\
\hline 25 & 2 & 1 & 2 & 32 & 12.5 & 6.2 & $<0.1$ & 0.8 & 12.5 & 1.6 \\
\hline 26 & 4 & 1 & 2 & 64 & 25 & 12.5 & $\mathrm{NG}$ & 1.6 & 6.2 & 0.2 \\
\hline 27 & 0.5 & 0.1 & 0.1 & 4 & 12.5 & 6.2 & 0.4 & 0.8 & 12.5 & 0.8 \\
\hline 28 & 0.5 & 0.2 & 0.5 & 16 & 12.5 & 12.5 & 0.1 & 0.8 & 6.2 & 0.4 \\
\hline 29 & 0.2 & 0.2 & 0.5 & 8 & 25 & 12.5 & 0.2 & 0.8 & 6.2 & 0.4 \\
\hline 30 & 0.5 & 0.2 & 1 & 16 & 12.5 & 6.2 & 0.1 & 0.4 & 12.5 & 0.8 \\
\hline 31 & 1 & 0.5 & 2 & 16 & 12.5 & 6.2 & 0.2 & 0.8 & 12.5 & 0.8 \\
\hline 32 & 0.5 & 0.5 & 1 & 16 & 25 & 50 & 0.4 & 1.6 & 6.2 & 0.4 \\
\hline 33 & 1 & 0.5 & 1 & 32 & 12.5 & 25 & NG & 0.4 & 6.2 & 0.8 \\
\hline 34 & 1 & 0.5 & 0.5 & 16 & 12.5 & 25 & NG & 0.4 & 6.2 & 0.1 \\
\hline 35 & 0.5 & 0.5 & 1 & 32 & 25 & 50 & $<0.1$ & 0.8 & 25 & 1.6 \\
\hline 36 & 1 & 0.5 & 1 & 32 & 12.5 & 12.5 & 0.2 & 3.1 & 50 & 0.8 \\
\hline 37 & 0.5 & 0.2 & 1 & 8 & 6.2 & 3.1 & 0.1 & 0.4 & 12.5 & 3.1 \\
\hline 38 & 0.5 & 0.2 & 0.2 & 16 & 6.2 & 3.1 & 3.1 & 0.8 & $>50$ & 12.5 \\
\hline 39 & 0.5 & 0.5 & 1 & 32 & 12.5 & 12.5 & 0.8 & 0.4 & 12.5 & 1.6 \\
\hline 40 & 0.2 & 0.2 & 0.2 & 4 & 6.2 & 3.1 & 1.6 & 0.8 & 12.5 & 1.6 \\
\hline 41 & 1 & 0.5 & 0.5 & 32 & 25 & 25 & 0.2 & 0.8 & 50 & 1.6 \\
\hline 42 & 0.5 & 0.2 & 0.2 & 8 & 3.1 & 6.2 & 0.4 & $<0.1$ & 50 & 0.8 \\
\hline 43 & 0.5 & 0.2 & 0.2 & 32 & 12.5 & 12.5 & $<0.1$ & 0.8 & 25 & 0.8 \\
\hline 44 & 0.5 & 0.5 & 2 & 64 & 25 & 25 & 0.2 & 3.1 & 25 & 0.8 \\
\hline 45 & 16 & 8 & 64 & $>128$ & $>50$ & $>50$ & 25 & 12.5 & $>50$ & 6.2 \\
\hline 46 & 2 & 1 & 8 & 128 & $>50$ & 50 & 1.6 & 1.6 & 12.5 & 3.1 \\
\hline 47 & 1 & 1 & 8 & 64 & 50 & 50 & 1.6 & 0.4 & 50 & $\mathrm{NG}$ \\
\hline 48 & 8 & 8 & 64 & $>128$ & $>50$ & 50 & 6.2 & 12.5 & $>50$ & $>25$ \\
\hline 49 & 1 & 0.5 & 0.5 & 128 & $>50$ & 50 & 0.2 & 0.2 & 3.1 & 0.2 \\
\hline 50 & 8 & 2 & 2 & 128 & $>50$ & $>50$ & 6.2 & 12.5 & $\mathrm{NG}$ & 6.2 \\
\hline 51 & 0.5 & 0.5 & 1 & 32 & 6.2 & 3.1 & 0.4 & 3.1 & $N G$ & 3.1 \\
\hline 52 & 32 & 128 & 16 & 128 & 50 & 50 & 50 & 25 & $>50$ & $N G$ \\
\hline 53 & 8 & 4 & 2 & 2 & 3.1 & 6.2 & 3.1 & 12.5 & 50 & NG \\
\hline 54 & 4 & 2 & 1 & 8 & 3.1 & 6.2 & 1.6 & 3.1 & $>50$ & NG \\
\hline
\end{tabular}


Table 3. (Continued)

\begin{tabular}{|c|c|c|c|c|c|c|c|c|c|c|}
\hline \multirow{2}{*}{ Compound } & \multicolumn{10}{|c|}{$\operatorname{MIC}(\mu \mathrm{g} / \mathrm{ml})^{\mathrm{a}}$} \\
\hline & S. $a$. & S. py. & S. pn. & $H . i$ & P. $m$. & P.h. & $M . g$. & $M . s$. & $M . h r$ & M. hp. \\
\hline 55 & 32 & 32 & 32 & 16 & 25 & 50 & 50 & 50 & 50 & NG \\
\hline 56 & 4 & 2 & 1 & 32 & 25 & 25 & 1.6 & 6.2 & 50 & 1.6 \\
\hline 57 & 4 & 2 & 8 & 128 & $>50$ & 50 & 3.1 & 0.8 & $>50$ & 12.5 \\
\hline 58 & 4 & 4 & 16 & 128 & 50 & 50 & 1.6 & 1.6 & $>50$ & 25 \\
\hline 59 & 0.5 & 0.2 & 0.5 & 32 & 25 & 50 & 0.4 & $<0.1$ & 1.6 & $<0.1$ \\
\hline 60 & 4 & 4 & 2 & 128 & $>50$ & 50 & 6.2 & 25 & $>50$ & 12.5 \\
\hline 61 & 4 & 4 & 2 & 64 & $>50$ & $>50$ & 3.1 & 3.1 & $N G$ & 0.8 \\
\hline 62 & 1 & 0.5 & 0.5 & 32 & 6.2 & 6.2 & 6.2 & 3.1 & 50 & 0.4 \\
\hline 63 & 2 & 1 & 2 & 64 & 12.5 & 12.5 & 6.2 & 12.5 & $N G$ & 1.6 \\
\hline 64 & 4 & 2 & 2 & 8 & 1.6 & 3.1 & 12.5 & 25 & 50 & 12.5 \\
\hline 65 & 2 & 2 & 0.2 & 8 & 3.1 & 0.8 & 3.1 & 12.5 & $>50$ & 3.1 \\
\hline 66 & 0.2 & 0.2 & $<0.1$ & 0.5 & 1.6 & 1.6 & 1.6 & NG & $\mathrm{NG}$ & 0.2 \\
\hline 67 & 4 & 1 & 0.5 & 16 & 12.5 & 12.5 & 3.1 & 6.2 & 50 & 1.6 \\
\hline 68 & 4 & 8 & 0.2 & 16 & 12.5 & 6.2 & 3.1 & NG & $\mathrm{NG}$ & 50 \\
\hline 69 & 1 & 2 & 0.2 & 4 & 3.1 & 1.6 & 6.2 & 6.2 & $>50$ & 6.2 \\
\hline 70 & 0.5 & 0.2 & 0.2 & 16 & 25 & 50 & 0.4 & 0.1 & 1.6 & 0.2 \\
\hline 71 & 0.5 & 0.2 & 0.2 & 4 & 6.2 & 25 & 0.8 & 0.2 & 3.1 & 0.2 \\
\hline 72 & 1 & 0.2 & 0.2 & 2 & 1.6 & 6.2 & 0.4 & 0.8 & 3.1 & 0.1 \\
\hline
\end{tabular}

a Methodology and organisms: Agar-dilution MIC values for: S. a., Staphylococcus aureus X1; S. py., Streptococcus pyogenes C 203; S. pn., Streptococcus pneumoniae Park 1; H. i., Haemophilus influenzae CL. Microtiter MIC values for: P. m., Pasteurella multocida, representative MIC of 5 strains; P. h., Pasteurella haemolytica, representative MIC of 3 strains; $M . g .$, Mycoplasma gallisepticum 29C; M. s., Mycoplasma synoviae 40A; $M$. hr., Mycoplasma hyorhinis 29E; M. hp., Mycoplasma hyopneumoniae $\mathrm{S} 5972$.

NG: Organism did not grow on day of test.

group (compounds $2,11,45,48,52$ and 55 ) were among the least active compounds tested. Activity was improved as the size of the $20-\mathrm{N}$-alkyl substituents increased beyond that of methyl, ethyl and small branched alkyl groups (such as found in compounds 3, 12,14 and 18). An upper limit to the size of the $\mathrm{N}$-alkyl substituents was not very well defined in terms of in vitro activity, which was only moderately reduced even with derivatives possessing such large C-20 substituents as (norbornyl)dodecylamino and (cyclooctyl)dodecylamino (compounds 25 and 26).

The demycarosyl derivatives of tylosin and related macrolides are known to have improved activity in vitro against Gram-negative bacteria, including the important veterinary pathogens $P$. multocida and $P$. haemolytica ${ }^{4,13}$. A similar trend was observed with the reductively aminated derivatives; these C-20 amino derivatives of tylosin, macrocin and $3^{\prime \prime \prime}-O$-demethylmacrocin (DOMM) had weak activity against Pasteurella species, whereas the analogous demycarosyl derivatives from desmycosin, lactenocin, 3"-O-demethyllactenocin (DOML) and OMT possessed good in vitro activity against both Pasteurella species (Table 3). Consequently, our principal effort was focused upon the demycarosyl derivatives as offering the best chance to significantly expand the useful antimicrobial spectrum of tylosin.

The optimum in vitro activity was achieved in those derivatives of desmycosin which possessed C-20 aminoalkyl substituents having approximately six to fourteen carbon atoms. Within this range, the aminoalkyl group could either be cyclic or acyclic, saturated or unsaturated (with double bonds, triple bonds or aromatic rings) or contain additional heteroatoms such as nitrogen, oxygen, sulfur or halogen. However, it was not possible to distinguish much further among the members of this relatively large group on the basis of their in vitro activity. 
Table 4. In vivo antimicrobial efficacy of derivatives from reductive amination of tylosin and related macrolides.

\begin{tabular}{|c|c|c|c|c|c|}
\hline \multirow{2}{*}{ Compound } & \multicolumn{2}{|c|}{$\begin{array}{c}\mathrm{ED}_{50} \text { values against } \\
\text { Streptococcus pyogenes in mice }\end{array}$} & \multicolumn{3}{|c|}{$\begin{array}{c}\text { Mortality in chicks } \\
\text { infected by Pasteurella multocida }{ }^{\mathrm{a}}\end{array}$} \\
\hline & $\mathrm{sc}^{\mathrm{b}}$ & $\mathrm{po}^{\mathrm{b}}$ & $s c^{c}$ & po $(0.53 \mathrm{~g} / \text { liter })^{d}$ & po $(0.26 \mathrm{~g} / \text { liter })^{\mathrm{d}}$ \\
\hline 1 & $>10$ & $>100$ & $\mathrm{Nt}$ & & \\
\hline 2 & 4.0 & $>100$ & $\mathrm{Nt}$ & & \\
\hline 3 & 0.6 & 44 & $\mathrm{Nt}$ & & \\
\hline 4 & $<0.6$ & 22 & Nit & & \\
\hline 5 & $<0.6$ & 70 & $\mathrm{Nt}$ & $8 / 10$ & $7 / 10$ \\
\hline 6 & $<1.6$ & 39 & $0 / 10$ & $10 / 10$ & \\
\hline 7 & $<1.6$ & 55 & $2 / 10$ & $10 / 10$ & \\
\hline 8 & 0.9 & 62 & $4 / 10$ & $6 / 10$ & \\
\hline 9 & 1.1 & 18 & $6 / 10$ & $5 / 10$ & $10 / 10$ \\
\hline 10 & 3.4 & $>100$ & $10 / 10$ & & \\
\hline 11 & $<0.6$ & $>100$ & $\mathrm{Nt}$ & $7 / 10$ & $8 / 10$ \\
\hline 12 & 1.7 & 71 & $\mathrm{Nt}$ & $7 / 10$ & $6 / 10$ \\
\hline 13 & 1.0 & 41 & $0 / 10$ & $1 / 10$ & $4 / 10$ \\
\hline 14 & 2.0 & 77 & $0 / 10$ & $8 / 10$ & \\
\hline 15 & 1.2 & 25 & $1 / 10$ & $4 / 10$ & $9 / 10$ \\
\hline 16 & 3.4 & 10 & $5 / 10$ & $6 / 10$ & $9 / 10$ \\
\hline 17 & 1.1 & 28 & $0 / 10$ & $2 / 10$ & $4 / 10$ \\
\hline 18 & 1.0 & 64 & $0 / 10$ & $4 / 10$ & $9 / 10$ \\
\hline 19 & 1.0 & 43 & $0 / 10$ & $1 / 10$ & $5 / 10$ \\
\hline 20 & 0.8 & 27 & $0 / 10$ & $6 / 10$ & $7 / 10$ \\
\hline 21 & 7.5 & 54 & $10 / 10$ & $7 / 10$ & $6 / 10$ \\
\hline 22 & 4.5 & 31 & $7 / 10$ & $4 / 10$ & $10 / 10$ \\
\hline 23 & 6.6 & 35 & $10 / 10$ & $10 / 10$ & \\
\hline 24 & 10.0 & 100 & $10 / 10$ & & \\
\hline 25 & $>10$ & $>100$ & $10 / 10$ & & \\
\hline 26 & $>10$ & $>100$ & $10 / 10$ & & \\
\hline 27 & 0.8 & 10 & $1 / 10$ & $4 / 10$ & $6 / 10$ \\
\hline 28 & 2.1 & 20 & $2 / 10$ & $9 / 10$ & $6 / 10$ \\
\hline 29 & 1.7 & 28 & $4 / 10$ & $8 / 10$ & $9 / 10$ \\
\hline 30 & 1.7 & 7 & $5 / 10$ & $5 / 10$ & $9 / 10$ \\
\hline 31 & 1.7 & 8 & $7 / 10$ & $3 / 10$ & $4 / 10$ \\
\hline 32 & 2.0 & 35 & $\mathrm{Nt}$ & & \\
\hline 33 & 3.2 & 18 & $10 / 10$ & & \\
\hline 34 & 2.3 & 45 & $8 / 10$ & & \\
\hline 35 & $>10$ & $>50$ & $8 / 10$ & $6 / 10$ & \\
\hline 36 & 2.3 & $>100$ & $1 / 10$ & $10 / 10$ & $10 / 10$ \\
\hline 37 & 1.4 & 15 & $6 / 10$ & $8 / 10$ & $9 / 10$ \\
\hline 38 & 0.9 & 50 & $0 / 10$ & $5 / 10$ & \\
\hline 39 & 2.0 & 35 & $10 / 10$ & & \\
\hline 40 & 1.4 & 18 & $2 / 10$ & $0 / 10$ & $3 / 10$ \\
\hline 41 & 6.5 & 35 & $10 / 10$ & $7 / 10$ & \\
\hline 42 & 6.0 & 20 & $9 / 10$ & $6 / 10$ & \\
\hline 43 & 5.6 & 13 & $10 / 10$ & & \\
\hline 44 & 8.7 & 46 & $6 / 10$ & $7 / 10$ & $10 / 10$ \\
\hline 45 & 7.4 & $>100$ & $\mathrm{Nt}$ & & \\
\hline 46 & 8.2 & 62 & $\mathrm{Nt}$ & & \\
\hline 47 & 9.1 & 76 & $\mathrm{Nt}$ & & \\
\hline 48 & 10.0 & $>100$ & $\mathrm{Nt}$ & & \\
\hline 49 & $>10$ & 76 & $\mathrm{Nt}$ & & \\
\hline 50 & 9.2 & $>100$ & $\mathrm{Nt}$ & & \\
\hline 51 & $>10$ & $>100$ & $10 / 10$ & $10 / 10$ & \\
\hline 53 & 16.1 & $>100$ & $1 / 10$ & & \\
\hline 54 & 7.2 & $>100$ & $1 / 10$ & $10 / 10$ & \\
\hline
\end{tabular}


Table 4. (Continued)

\begin{tabular}{|c|c|c|c|c|c|}
\hline \multirow{2}{*}{ Compound } & \multicolumn{2}{|c|}{$\begin{array}{c}\mathrm{ED}_{50} \text { values against } \\
\text { Streptococcus pyogenes in mice }\end{array}$} & \multicolumn{3}{|c|}{$\begin{array}{c}\text { Mortality in chicks } \\
\text { infected by Pasteurella multocida }\end{array}$} \\
\hline & $s c^{b}$ & $\mathrm{po}^{\mathrm{b}}$ & $\mathrm{sc}^{\mathrm{c}}$ & po $\left(0.53 \mathrm{~g} /\right.$ liter $^{\mathrm{d}}$ & po $(0.26 \mathrm{~g} / \text { /iter })^{\mathrm{d}}$ \\
\hline 57 & $>10$ & 44 & $\mathrm{Nt}$ & & \\
\hline 58 & $>10$ & 30 & $\mathrm{Nt}$ & & \\
\hline 59 & 1.9 & 27 & $\mathrm{Nt}$ & & \\
\hline 60 & $>10$ & $>100$ & $\mathrm{Nt}$ & & \\
\hline 61 & 4.4 & $>100$ & $\mathrm{Nt}$ & & \\
\hline 62 & 1.8 & 100 & $0 / 10$ & & \\
\hline 63 & 8.2 & $>100$ & $0 / 10$ & $9 / 10$ & \\
\hline 64 & 7.8 & $>100$ & $0 / 10$ & $10 / 10$ & \\
\hline 65 & $>10$ & $>100$ & $\mathrm{Nt}$ & $10 / 10$ & \\
\hline 66 & 2.9 & $>100$ & $4 / 10$ & & \\
\hline 67 & $>10$ & $>100$ & $\mathrm{Nt}$ & & \\
\hline 68 & 10 & $>100$ & $\mathrm{Nt}$ & & \\
\hline 69 & 5.9 & 87 & $1 / 10$ & $7 / 10$ & \\
\hline 70 & 1.3 & 42 & $8 / 10$ & & \\
\hline 71 & 1.4 & 84 & $0 / 10$ & $8 / 10$ & $8 / 10$ \\
\hline 72 & 3.9 & 102 & $0 / 10$ & $10 / 10$ & $10 / 10$ \\
\hline
\end{tabular}

a Number of chicks which died per number which were medicated.

b $\mathrm{ED}_{50}$ values given in $\mathrm{mg} / \mathrm{kg} \times 2$, given 1 and 5 hours post-infection.

c Compound administered at $30 \mathrm{mg} / \mathrm{kg}$, given 1 and 4 hours post-infection.

d Compound administered at specified concentration in chicks' drinking water.

Nt: Not tested in vivo.

\section{In Vivo Activity}

Because it was difficult to choose from the many members of this series of derivatives on the basis of their in vitro activity, their in vivo activity became the critical parameter for selecting the potentially most useful antimicrobial compounds. In vivo activity was initially assessed in two different model experimental infections. Efficacy against a typically susceptible Gram-positive bacterium, Streptococcus pyogenes, was measured in a traditional mouse infection model, whereas efficacy against $P$. multocida was determined in chicks, due to the greater reproducibility of our results in this experimental infection model ${ }^{4}$. Compounds were generally tested by both the parenteral and oral routes, since antimicrobial efficacy by both routes of administration was being sought.

As reported previously, the $\mathrm{C}-20$ modified derivatives of desmycosin were generally efficacious against infections by Gram-positive bacteria in mice and, in particular, were more efficacious than tylosin or desmycosin when administered orally ${ }^{3}$. A substantial number of the C-20 aminoalkyl derivatives of desmycosin followed this same pattern, exhibiting good efficacy when administered orally against the experimental infection by $S$. pyogenes (Table 4). The C-20 aminoalkyl derivatives possessing either small substituents (compounds 1, 2, 11, 45 and 48) or large substituents (compounds 10, 24, 25 and 26) were not orally active, which helped define the range of compounds with potentially useful oral efficacy. The C-20 aminoalkyl derivatives of OMT (compounds $52 \sim 56$ and $64 \sim 69$ ) were all inactive when given orally, in spite of their good in vitro activity and, in many cases, their good in vivo activity when given parenterally.

In vivo activity of the derivatives against Pasteurella was investigated in a chick infection model to further define the range of effective antibiotic compounds. In this preliminary in vivo screen, a dose of $30 \mathrm{mg} / \mathrm{kg}$ was used subcutaneously while an oral dose of $0.53 \mathrm{~g} /$ liter was administered in the drinking water of the chicks. All compounds with an MIC value against Pasteurella of $6.25 \mu \mathrm{g} / \mathrm{ml}$ or less, as 
well as some derivatives with higher MIC values, were evaluated in vivo. Based upon results from the preliminary in vivo tests, dose titration studies were then performed to select the compounds with the best in vivo activity. Although an inevitable amount of test-to-test variability was present, this experimental infection provided a suitable basis for selecting the most efficacious compounds.

This chick Pasteurella test represented a relatively severe infection and consequently posed a serious challenge to the compounds for demonstrating in vivo activity. While a number of the reductive amination derivatives, when given subcutaneously, treated the Pasteurella infection quite well at $30 \mathrm{mg} / \mathrm{kg}$ or even at lower doses, very few compounds clearly stood out as efficacious when administered orally in the drinking water of the chicks. Among the acyclic aminoalkyl compounds, the better candidates appeared to be those tertiary amino derivatives with alkyl groups of three to five carbon atoms in length, such as the di(n-propyl)amino, the di(sec-butyl)amino and the ethyl(2-methylbutyl)amino derivatives of desmycosin (compounds 13, 17 and 19). In contrast, many of the cyclic aminoalkyl derivatives, exemplified by the 3,5-dimethylpiperidinyl derivative (40), appeared to be somewhat more reproducibly efficacious in this model Pasteurella infection in chicks and have been reported separately ${ }^{12)}$.

As previously reported for other aldehyde-modified derivatives of desmycosin ${ }^{3)}$, the derivatives obtained by reductive amination of desmycosin gave higher blood levels than either desmycosin or tylosin in both rats and chicks after oral administration (Table 5). Furthermore, higher concentrations were achieved more consistently in chicks than in rats. The combination of excellent efficacy in two distinctly different models of experimental infections, utilizing two different animal species and two different bacteria, along with results demonstrating good bioavailability after oral administration, indicated that a useful new oral antibiotic could be selected from this series of reductive amination derivatives of desmycosin.

In order to better distinguish among the number of these derivatives which appeared to exhibit the desired characteristics, a smaller group was selected for further evaluation in the target animals, calves and pigs. Included from the present series as the best candidates were the di( $n$-propyl)amino derivative (compound 13) and the ethyl(2-methylbutyl)amino derivative (compound 19) of desmycosin, along with representatives of the cyclic aminoalkyl derivatives, as exemplified by the 3,5-dimethylpiperidinyl derivative

Table 5. Peripheral blood concentrations of reductive amination derivatives at $100 \mathrm{mg} / \mathrm{kg}$ orally in rats and chicks.

\begin{tabular}{|c|c|c|c|c|c|c|c|}
\hline \multirow{2}{*}{ Compound } & \multirow{2}{*}{ Animal } & \multicolumn{6}{|c|}{ Mean concentration $(\mu \mathrm{g} / \mathrm{ml})$ per sample period } \\
\hline & & 0.25 hour & 0.5 hour & 1.0 hour & 2.0 hours & 4.0 hours & 6.0 hours \\
\hline \multirow[t]{2}{*}{ Tylosin } & Rat & 0 & 0 & 0 & 0 & 0 & 0 \\
\hline & Chick & 0.3 & 0.7 & 2.8 & 1.7 & 0 & 0 \\
\hline \multirow[t]{2}{*}{ Desmycosin } & Rat & 0 & 0 & 0 & 0 & 0 & 0 \\
\hline & Chick & 0 & 0 & 0.1 & 4.4 & 一 & 一 \\
\hline \multirow{2}{*}{$\begin{array}{l}\text { 20-Deoxo-20-(hexamethyleneimino)- } \\
\text { desmycosin }\end{array}$} & Rat & 0 & 0 & 0 & 0 & 0 & 0 \\
\hline & Chick & 16.4 & 19.8 & 16.3 & 28.4 & 13.2 & 8.8 \\
\hline \multirow{2}{*}{$\begin{array}{l}\text { 20-Deoxo-20-(heptamethyleneimino)- } \\
\text { desmycosin }{ }^{a}\end{array}$} & Rat & 0 & 0 & 0 & 3.3 & 2.1 & 1.6 \\
\hline & Chick & 3.5 & 2.4 & 4.0 & 3.2 & 1.8 & 1.8 \\
\hline \multirow{2}{*}{$\begin{array}{l}\text { 20-Deoxo-20-(dodecamethyleneimino)- } \\
\text { desmycosin }\end{array}$} & Rat & 1.4 & 2.2 & 1.6 & 3.7 & 5.0 & 4.6 \\
\hline & Chick & 2.5 & 5.6 & 7.8 & 9.0 & 11.2 & 8.2 \\
\hline \multirow{2}{*}{$\begin{array}{l}\text { 20-Deoxo-20-(3-azabicyclo[3.2.2]nonanyl)- } \\
\text { desmycosin }\end{array}$} & Rat & 0 & 0 & 0 & 2.6 & 2.5 & 1.5 \\
\hline & Chick & 0.4 & 9.0 & 26.2 & 16.4 & 9.8 & 8.9 \\
\hline \multirow{2}{*}{$\begin{array}{l}\text { 20-Deoxo-20-(4-phenylpiperidinyl)- } \\
\text { desmycosin }\end{array}$} & Rat & 1.0 & 1.7 & 2.5 & 3.5 & 3.6 & 0 \\
\hline & Chick & 3.8 & 12.0 & 9.5 & 11.1 & 20.1 & 14.6 \\
\hline
\end{tabular}

a See ref 12 . 
(compound 40) of desmycosin. From these more extensive investigations, the latter compound (40) was selected as the best candidate for further development. Now generically known as tilmicosin (originally designated as EL-870), it is being investigated for the treatment of pneumonia in cattle and pigs ${ }^{6,14,15)}$.

\section{Experimental}

Materials and Methods

${ }^{1} \mathrm{H}$ NMR spectra were measured in $\mathrm{CDCl}_{3}$ solution on a Bruker WH-360 or Jeol FX90A spectrometer; chemical shifts are given in ppm downfield from internal TMS. Field desorption mass spectra (FD-MS) were obtained on a Varian-MAT 731 spectrometer with carbon dendrite emitters. UV spectra were measured in $95 \%$ ethanol solution on a Cary 219 spectrometer. TLC was performed using E. Merck plates of Silica gel 60 with a fluorescent indicator (F-254); visualization was effected by UV light. Product purification was performed by chromatography on silica gel, using either flash chromatography ${ }^{16)}(\mathrm{E}$. Merck grade 60 silica gel) or a Waters Model 500 Prep LC system.

In vivo evaluations against $S$. pyogenes infections in mice were conducted by treating infected animals 1 and 5 hours post-infection, either subcutaneously or by gavage, with $0.25 \mathrm{ml}$ of a $10 \%$ aqueous ethanolic solution of the compound over a range of concentrations; tartaric acid was added when needed to dissolve the compounds. In vivo evaluations against $P$. multocida were performed by treating infected 1-day-old chicks with the test compound, either subcutaneously at 1 and 4 hours post-infection or orally by dissolution of the compound in the drinking water of the animals, provided ad libitum. Peripheral plasma concentrations were determined by microbiological assay, using Micrococcus luteus seeded in Difco Antibiotic Media 1. Concentrations represent an average value of 5 mice per time period or 6 chicks per time period.

\section{0-Deoxo-20-(diisobutylamino)desmycosin (16)}

Desmycosin $(3.0 \mathrm{~g}, 3.9 \mathrm{mmol})$ was dissolved in methanol $(30 \mathrm{ml})$ in the presence of $3 \mathrm{~A}$ molecular sieves. Diisobutylamine $(1.5 \mathrm{~g}, 11.7 \mathrm{mmol})$ was added, and after 15 minutes, sodium cyanoborohydride $(0.74 \mathrm{~g}$, $11.7 \mathrm{mmol}$ ) was added. After being stirred at room temperature for 17 hours, the mixture was filtered, and the filtrate was evaporated to dryness. The resultant yellow solid foam was dissolved in ethyl acetate $(200 \mathrm{ml})$ and extracted with water $(200 \mathrm{ml})$; the product was then extracted into aqueous sodium dihydrogen phosphate buffer solution $(250 \mathrm{ml}, \mathrm{pH} 6.5,0.5 \mathrm{M})$. The aqueous solution was separated and residual ethyl acetate was evaporated under reduced pressure. The resultant solution was rapidly stirred while aqueous sodium hydroxide $(5 \mathrm{~N})$ was added to precipitate the desired product; final $\mathrm{pH}$ was approximately 8 . The white solid was filtered and dried to give $1.34 \mathrm{~g} \mathrm{(39 \% )} \mathrm{of} \mathrm{20-deoxo-20-(diisobutylamino)desmycosin:} \mathrm{See}$ Table 1 for MS, UV and NMR data; $\mathrm{p} K_{\mathrm{a}}(66 \% \mathrm{DMF}) 7.6$ and 9.0 .

\section{0-Deoxo-20-morpholinyldesmycosin (36)}

Desmycosin $(11.6 \mathrm{~g}, 15 \mathrm{mmol})$ and morpholine $(2.6 \mathrm{ml}, 30 \mathrm{mmol})$ were dissolved in methanol $(100 \mathrm{ml})$ and after 30 minutes, sodium cyanoborohydride $(1.25 \mathrm{~g}, 20 \mathrm{mmol})$ was added. After being stirred overnight at room temperature, solvent was evaporated under reduced pressure. The residue was partitioned between water $(150 \mathrm{ml})$ and ethyl acetate $(150 \mathrm{ml})$, the aqueous layer was separated, and the organic solution was extracted twice with aqueous sodium phosphate solution $(0.5 \mathrm{M}$, initial $\mathrm{pH} 6.5,75 \mathrm{ml}$ each). The combined aqueous layers were evaporated under reduced pressure to remove residual organic solvents and then treated with aqueous sodium hydroxide solution while being vigorously stirred until the $\mathrm{pH}$ had been raised to 10 . The solid clump of precipitate was extracted with ethyl acetate, and the organic solution was dried, filtered, and evaporated to yield $7.7 \mathrm{~g} \mathrm{(61 \% )}$ ) of the desired compound; $\mathrm{p} K_{\mathrm{a}}(66 \% \mathrm{DMF}) 6.5$ and 8.5 .

\section{0-Deoxo-20-neopentylaminodesmycosin (4)}

The reductive amination was performed as described above, using neopentylamine $(3.5 \mathrm{ml}, 30 \mathrm{mmol})$, to yield $8.8 \mathrm{~g}(70 \%)$ of the desired product: $\mathrm{p} K_{\mathrm{a}}(66 \% \mathrm{DMF}) 7.9$ and 9.7 . After the extraction with the pH 6.5 phosphate buffer solution, the organic solution was extracted with $\mathrm{pH} 5.5$ aqueous phosphate buffer solution which, upon analogous workup, yielded $1.4 \mathrm{~g}$ of a mixture of impurities, indicating the potential for selective purification of the desired products by appropriate extractive procedures. 


\section{0-Deoxo-20-(bicyclo[2.2.1]hept-2-yl)(n-octyl)aminodesmycosin (24)}

To a stirred solution of desmycosin $(8.0 \mathrm{~g}, 10.3 \mathrm{mmol})$ and (bicyclo[2.2.1]hept-2-yl)(n-octyl)amine $(2.5 \mathrm{~g}, 11.1 \mathrm{mmol})$ in dry methanol $(50 \mathrm{ml})$ was added sodium cyanoborohydride $(0.7 \mathrm{~g}, 11 \mathrm{mmol})$ in portions over a five-minute period. The solution was stirred overnight at room temperature and solvent was then evaporated under reduced pressure. The residue was dissolved in ethyl acetate $(100 \mathrm{ml})$ and extracted first with water $(100 \mathrm{ml})$ and then with $5 \%$ aqueous sodium dihydrogen phosphate solution $(100 \mathrm{ml})$. The aqueous acidic extract $(\mathrm{pH} 4)$ was adjusted first to $\mathrm{pH} 6$ with $6 \mathrm{~N}$ sodium hydroxide and extracted with ethyl acetate, then adjusted to $\mathrm{pH} 7$ and extracted with ethyl acetate, and finally adjusted to $\mathrm{pH} 8$ and extracted as before. The organic extract from the $\mathrm{pH} 7$ aqueous solution was dried (magnesium sulfate) and filtered and the filtrate was evaporated under reduced pressure to yield $4.4 \mathrm{~g}(45 \%)$ of the title compound.

\section{Acknowledgments}

We thank J. PASCHal and associates for NMR spectra, J. Occolowitz and associates for MS, A. HuNT and associates for UV spectra, A. Kossoy and associates for $\mathrm{p} K_{\mathrm{a}}$ measurements, L. HuCKSTEP and R. THomas for preparative HPlC separations, and S. A. Stroy, H. M. Michael, M. D. Newport, P. Lubbehusen, G. Murphy, M. Staples and C. Cummins for technical assistance in evaluation experiments. We are indebted to Dr. G. M. WILD for helpful discussions as well as generous supplies of starting materials.

\section{References}

1) Wilson, R. C.: Chapter 8. Macrolides in veterinary practice. In Macrolide Antibiotics. Chemistry, Biology, and Practice. Ed., S. ŌMURA, pp. $301 \sim 347$, Academic Press, 1984

2) LANGWORTH, B. F.: Fusobacterium necrophorum: Its characteristics and role as an animal pathogen. Bacteriological Rev. 41: 373 390, 1977

3) Kirst, H. A.; J. E. Toth, M. Debono, K. E. Willard, B. A. Truedell, J. L. Otr, F. T. Counter, A. M. FELTY-Duckworth \& R. S. PEKAREK: Synthesis and evaluation of tylosin-related macrolides modified at the aldehyde function: A new series of orally effective antibiotics. J. Med. Chem. 31: 1631 1641, 1988

4) Kirst, H. A.; E. E. Ose, J. E. Toth, K. E. Willard, M. Debono, A. M. Felty-Duckworth \& R. S. PeKarek: In vitro and in vivo evaluation of $\mathrm{C}-20$ - and $\mathrm{C}-23$-modified derivatives of tylosin against veterinary pathogens. $\mathrm{J}$. Antibiotics 41: 938 948, 1988

5) Wohlgemuth, K. \& J. B. Herrick: Bovine respiratory disease complex, an overview. Animal Health \& Nutrition 41: $26 \sim 28,1986$

6) OSE, E. E. \& L. V. TONKINSON: Tilmicosin in feed for the control of induced pneumonic pasteurellosis in pigs. In Proceedings of the 10th International Pig Veterinary Society Congress. Chapter 3, Scientific Committee of the 10th Congress, p. 62, Brazil, Aug. 14 17, 1988

7) Sakakibara, H. \& S. Ōmura: Chapter 3. Chemical modification and structure-activity relationship of macrolides. In Macrolide Antibiotics. Chemistry, Biology, and Practice. Ed., S. OMURA, pp. 85 125, Academic Press, 1984

8) Ōmura, S.; K. MiYano, H. Matsubara \& A. NaKagawa: Novel dimeric derivatives of leucomycins and tylosin, sixteen-membered macrolides. J. Med. Chem. 25: $271 \sim 275,1982$

9) Matsubara, H.; J. Inokoshi, A. Nakagawa, H. Tanaka \& S. OMura: Chemical modification of tylosin: Synthesis of amino derivatives at C-20 position of tylosin and demycarosyltylosin. J. Antibiotics 36: 1713 1721, 1983

10) Tanaka, A.; T. Tsuchiya, Y. OKada, S. Umezawa, M. Hamada \& H. Ụmzawa: Syntheses of 23-dialkylamino derivatives of mycaminosyl tylonolide and 4'-deoxy-mycaminosyl tylonolide effective against Gram-negative bacteria. J. Antibiotics 35: $113 \sim 116,1982$

11) Borch, R. F.; M. D. Bernstein \& H. D. Durst: The cyanohydridoborate anion as a selective reducing agent. J. Am. Chem. Soc. 93: 2897 2904, 1971

12) Debono, M.; K. E. Willard, H. A. Kirst, J. A. Wind, G. D. Crouse, E. V. Tao, J. T. Vincenzi, F. T. Counter, J. L. OTT, E. E. OSE \& S. OMURA: Synthesis and antimicrobial evaluation of 20-deoxo-20-(3,5-dimethylpiperidin1-yl)desmycosin (tilmicosin, EL-870) and related cyclic amino derivatives. J. Antibiotics 42: 1253 1267, 1989

13) Kirst, H. A.; G. M. Wild, R. H. Baltz, R. L. Hamill, J. L. Ott, F. T. Counter \& E. E. Ose: Structure-activity studies among 16-membered macrolide antibiotics related to tylosin. J. Antibiotics 35: 1675 1682, 1982

14) OSE, E. E.: In vitro antibacterial properties of EL-870, a new semi-synthetic macrolide antibiotic. J. Antibiotics 40: $190 \sim 194,1987$

15) OSE, E. E. \& L. V. Tonkinson: Single-dose treatment of neonatal calf pneumonia with the new macrolide antibiotic tilmicosin. The Veterinary Record 123: 367 369, 1988

16) StILl, W. C.; KAHN \& A. MITRA: Rapid chromatographic technique for preparative separations with moderate resolution. J. Org. Chem. 43: $2923 \sim 2925,1978$ 